\title{
Action of galvanic currents on needles during percutaneous needle electrolysis
}

\author{
Margalef R. ${ }^{1} \quad$ Minaya Muñoz F. ${ }^{2} \quad$ Valera Garrido F. ${ }^{2} \quad$ Santafe M. M. ${ }^{1}$ \\ 1 Unit of Histology and Neurobiology, Department of Basic Medical \\ Sciences, Faculty of Medicine and Health Sciences, Rovira i Virgili \\ University, Reus, Spain \\ 2 Servicio de Fisioterapia MVClinic. Pozuelo de Alarcón, Madrid, Spain \\ Rev Fisioter Invasiva 2019;2:110-111.
}

\begin{abstract}
Keywords

- Galvanic currents

- percutaneous needle electrolysis

- electric resistance

- temperature

- electronic scanning microscope

- microanalysis
\end{abstract}

Background The use of galvanic currents (GC), during percutaneous needle electrolysis (PNE) can make needles release metals and therefore decrease their diameter and increase resistance. If this were very marked, this could increase the temperature of the needle during use of the same. Furthermore, the loss of materials such as zinc, can trigger allergic reactions in people who are sensitive to these metals.

Aim to evaluate modifications of needles during the application of GC in PNE. Methods The resistance was evaluated using a Digital multimeter Velleman DVM92. The GC was generated using the Physio Invasiva device by Prim ( $3 \mathrm{~mA}$ during 3 seconds, 3 applications were performed). For each needle, resistance of the proximal and distal area was evaluated (insertion) and the \% of variation was calculated. 20 Physio Invasiva Needles measuring $0.30 \times 40 \mathrm{~mm}$ were evaluated and inserted in mice. A Quante 600 scanningmicroscope was used as well as a microanalysis device based on diffusion by RX Oxford Instruments (Universidad Rovira i Virgili). The protocols used were: A) $3 \mathrm{~mA}$ during 3 seconds and 3 applications (Physio Invasiva by BRUMA Center-Tarragona ( 6 needles) and from the MV ClinicMadrid ( 9 needles) in humans and from the UHN laboratory UHN (10 needles) in animals; B) 3mA during 3 seconds and 10 applications, 5 needles were evaluated in animals; C) 3mA during 3 seconds and 50 applications, 5 needles were evaluated in animals.

Results No change was obtained in the proximal-distal resistance $(0.4 \% \pm 3.33)$. No loss of any of the metals occurred in the needles subjected to the protocol of $3 / 3 / 3$ nor the protocol $3 / 3 / 10$. However, in the $3 / 3 / 10$ protocol, statistically significant losses were obtained for all the metals present.

Conclusion Physio Invasiva needles measuring $0.30 \times 40 \mathrm{~mm}$ do not shed metals nor modify their morphology during the intervention, therefore, the needle diameter of the needle does not decrease. Only exceptionally, when the number of applications is large, is there a loss of material.
DOI https://doi.org/ $10.1055 / \mathrm{s}-0039-3401882$ ISSN 2386-4591.
Copyright ( 2019 by Thieme Revinter Publicações Ltda, Rio de Janeiro, Brazil
License terms 
Acknowledgments

The authors wish to thank Dr. Mariana Stankova of the Service of Scientific and Technical Resources of the URV for their unequable technical advice in the determinations of the electronic scanning microscope. In addition, we thank the company PRIM Fisioterpia who has selflessly provided the needles and the Physio Invasiva ${ }^{\circledR}$ device used in this study to create galvanic currents. Special thanks to the BRUMA center in Tarragona and MVClinic in Madrid for kindly providing the needles used in their daily practice. This research is partially funded by a grant by the Instituto de Salud Carlos III, Acción Estratégica en Salud (MMS, PI13 / 02084). 